\title{
ERROR RESILIENT MULTIPLE DESCRIPTION COMPRESSION OF VECTOR GRAPHICS
}

\author{
Martin Röder ${ }^{1}$, Xiaolin $W^{2}$, Sorina Dumitrescu ${ }^{2}$ \\ ${ }^{1}$ University of Konstanz, Germany; ${ }^{2}$ McMaster University, Canada
}

\begin{abstract}
This research is motivated by the needs of robust streaming of vector graphics contents over the Internet, wireless and other lossy networks. We present a multiple description coding (MDC) technique for error resilient compression and transmission of 2D vector graphics contents. An object is coded into two or more so-called co-descriptors, which are transmitted in separate data packets and generally via different network routes from a server to a client. Each co-descriptor can autonomously provide an approximation of the input object, and it can collaborate with other co-descriptors, if also available at the decoder, to refine the approximation.
\end{abstract}

\section{INTRODUCTION}

Polygonal curves and shapes are basic constructs of computer graphics, geographic information systems (GIS), computer aided design, object-based image/video compression, and computer vision. In the prevalence of digital multimedia communications via the Internet or wireless networks, vector graphics data are used in many real-time visual communication applications, such as real-time vehicle navigation, object-based video transmission, networked interactive computer games, just to name few. The transmission of vector graphics contents is often subject to hostile channel conditions and low and varying bandwidth. For instance, when a mobile GIS user requests an electronic map using a wireless device in an urban area, the transmission quality can degrade due to channel fading, and the data packets can be delayed due to network congestions, or even lost/corrupted due to channel errors. In such practical scenarios, error resilient compression of vector graphics data is crucial to quality of service (QoS).

A common technique of compressing graphics data is polygonal approximation of digital curves or shapes $[1,2]$. The goal is to fit a discrete curve to a given precision with an open or closed polygonal chain of the smallest possible number of edges. For further compression gains, a predictive coding of the sequence of the resulting polygonal segments is typically applied. The compressed geometric code stream is then packetized and transmitted over a lossy network. However, without error resilience measures the compressed digital curves are highly susceptible to transmission errors. If a data packet is lost or delayed beyond the deadline for using the data, the decoder may fail completely to reconstruct the curve in time. In order to prevent such a worst-case catastro- phe in QoS, we propose a multiple description coding (MDC) scheme for compression of $2 \mathrm{D}$ vector graphics data.

In MDC compression of vector graphics a digital curve is coded into two or more so-called co-descriptors, which are transmitted in separate data packets and generally via different network routes from a server to a client. Each codescriptor can be decoded independently and provides a coarse description of the input curve. If few or all co-descriptors are received, they can collaborate and refine the curve representation.

\section{PROBLEM FORMULATION}

2D geometry compression can be achieved by approximating a high resolution curve by a polygon or polygonal curve that has a much smaller number of vertices than in the original. For the ease and concreteness of presentation and without loss of generality, we formulate the MDC compression problem and develop our co-descriptor design algorithm for open polygonal curves.

An open $N$-vertex polygonal curve $\mathcal{C}$ is represented as an ordered set of vertices $\mathcal{C}=\left\{\mathbf{v}_{1}, \cdots, \mathbf{v}_{N}\right\}$. An $M$-vertex polygonal approximation of $\mathcal{C}$ is a subset $S=\left\{\mathbf{v}_{s_{1}}, \cdots, \mathbf{v}_{s_{M}}\right\}$ of $\mathcal{C}$, such that $1=s_{1}<\cdots<s_{M}=N$. Thus, the portion of the original curve between vertices $\mathbf{v}_{s_{m}}$ and $\mathbf{v}_{s_{m+1}}$ is approximated by the polygonal edge (linear segment) $\left(\mathbf{v}_{s_{m}}, \mathbf{v}_{s_{m+1}}\right)$. We denote by $d\left(s_{m}, s_{m+1}\right)$ the distortion incured by using the linear segment $\left(\mathbf{v}_{s_{m}}, \mathbf{v}_{s_{m+1}}\right)$ as approximation for the original curve between $\mathbf{v}_{s_{m}}$ and $\mathbf{v}_{s_{m+1}}$. The developments in the sequel are valid for additive geometry distortion measures where the total approximation error is the sum of the segment distortions (e.g. mean squared distance or error area [3]). The total approximation error of $S$ is $D(S)=\sum_{m=1}^{M-1} d\left(s_{m}, s_{m+1}\right)$. By $r\left(s_{m}, s_{m+1}\right)$, we denote the rate (number of bits) for transmitting the linear segment $\left(\mathbf{v}_{s_{m}}, \mathbf{v}_{s_{m+1}}\right)$. We can use any vertex encoding scheme as long as $r\left(s_{m}, s_{m+1}\right)$ does not depend on vertices other than $\mathbf{v}_{s_{m}}$ and $\mathbf{v}_{s_{m+1}}$. The total rate of $S$ is $R(S)=\sum_{m=1}^{M-1} r\left(s_{m}, s_{m+1}\right)$.

The curve $\mathcal{C}$ is to be compressed and transmitted to a client through $K$ separate channels. To this end, we generate $K$ polygonal curves $S_{1}, \ldots, S_{k}$, called co-descriptors of $\mathcal{C}$. To simplify the notations, we represent the co-descriptors $S_{i}$ by the sequence of vertex indices $S=\left(i_{1}, \cdots, i_{M_{i}}\right), 1 \leq i \leq K$, $1 \leq M_{i} \leq N$. Each co-descriptor can be coded separately and decoded autonomously to an approximation of $\mathcal{C}$. If more than one co-descriptor is received, we want to combine them 
to a so-called joint descriptor that significantly improves upon each single co-descriptor. Such a joint descriptor can be generated by interleaving the received co-descriptors according to the order of the vertices in the original curve $\mathcal{C}$.

Assume that the $K$ channels are independent of each other, with transmission rates $r_{1}, \ldots, r_{k}$, and probabilities of successful transmission $p_{1}, \ldots, p_{k}$. Let $\mathbf{f}=\left(f_{1}, \cdots, f_{K}\right) \in$ $\{0,1\}^{K}$ be a random $K$-dimensional binary vector such that $f_{k}=1$ if the $k^{t h}$ descriptor is available and $f_{k}=0$ otherwise. A realization of $\mathbf{f}$ encodes a particular channel condition, i.e., which co-descriptors are available and which are not. We call an instance of $\mathbf{f}$ a configuration. Since all channels are independent, the probability mass function of the configurations can be expressed by $P(\mathbf{f})=\prod_{f_{i}=1} p_{i} \prod_{f_{j}=0}\left(1-p_{j}\right)$. Geometrically, denote by $S(\mathbf{f})=\cup_{f_{k}=1,1 \leq k \leq K} S_{k}$ the polygonal curve formed by connecting the vertices of the available codescriptors in the order of the original curve, i.e., the joint descriptor of the available co-descriptors. The expected distortion of the co-descriptors $S_{1}, \ldots, S_{k}$ is

$$
\bar{D}\left(S_{1}, \cdots, S_{K}\right)=\sum_{\mathbf{f} \in\{0,1\}^{K}} P(\mathbf{f}) D(S(\mathbf{f})) .
$$

MDC geometry compression can be cast as the problem of minimizing $\bar{D}\left(S_{1}, \cdots, S_{K}\right)$ over all possible combinations of approximation polygonal curves $S_{1}, \ldots, S_{k}$, subject to $R\left(S_{1}\right) \leq r_{1}, \ldots, R\left(S_{k}\right) \leq r_{k}$. Note that because the contribution of the configuration $\mathbf{f}=(0, \ldots, 0)$ to the overall distortion is a constant regardless of the varying co-descriptors, we set $D(S(\mathbf{f}))=0$.

\section{CO-DESCRIPTOR DESIGN}

We first rewrite the problem in Lagrangian form. Given a Lagrange multiplier $\lambda_{i}$ for each co-descriptor $S_{i}$, we want to minimize the expected Lagrangian

$$
\bar{J}_{\lambda_{1}, \ldots, \lambda_{K}}\left(S_{1}, \cdots, S_{K}\right)=\bar{D}\left(S_{1}, \cdots, S_{K}\right)+\sum_{i=1}^{K} \lambda_{i} R\left(S_{i}\right) \text {. }
$$

Computing $\bar{D}\left(S_{1}, \cdots, S_{K}\right)$ by expanding the summation of (1) is not tractable, even for modest values of $K$. Evaluating (1) takes $O\left(2^{K} M\right)$ time, where $M=\sum_{k=1}^{K} M_{k}$, let alone the minimization of (2). However, for a fixed value of $\mathbf{f}, D(S(\mathbf{f}))$ is the sum of the distortions of the approximation line segments of the polygonal curve $S(\mathbf{f})$, weighted by $P(\mathbf{f})$. Therefore $\bar{D}\left(S_{1}, \cdots, S_{K}\right)$ can be written as a weighted sum of the distortions of all segments of $S(\mathbf{f})$ over all possible configurations $\mathbf{f}$.

We now enumerate all the approximating line segments over all possible $2^{K}$ configurations $\mathbf{f} \in\{0,1\}^{K}$. A segment $\left(i_{a}, j_{b}\right)$ can be on the polygonal curve $S(\mathbf{f})$ for some $\mathbf{f}$ only if there exists a vertex $j_{t}$ of $S_{j}, 1 \leq j \leq K$, such that $i_{a} \leq j_{t} \leq$ $i_{a+1}$, and in that case we have $b=\min \left\{t \mid i_{a} \leq j_{t} \leq i_{a+1}\right\}$. We call these segments $\left(i_{a}, j_{b}\right)$ permissible segments. Note that under the above definition any edge $\left(i_{a}, i_{a+1}\right)$ of $S_{i}$ is a permissible segment. Let $\Xi$ be the set of all permissible segments. One can rewrite (1) as

$$
\bar{D}\left(S_{1}, \cdots, S_{K}\right)=\sum_{\left(i_{a}, j_{b}\right) \in \Xi} w\left(i_{a}, j_{b}\right) d\left(i_{a}, j_{b}\right),
$$

where the weight $w\left(i_{a}, j_{b}\right)$ is a function of $S_{1}, \cdots, S_{K}$ and $P(\mathbf{f})$. We define a set $\Psi\left(i_{a}, j_{b}\right)$ with respect to each permissible segment $\left(i_{a}, j_{b}\right): \Psi\left(i_{a}, j_{b}\right)=\left\{k \mid(\exists t)\left(i_{a}<k_{t}<j_{b}\right) ; 1 \leq\right.$ $k \leq K\}$. It identifies all the co-descriptors that have one or more vertices in between $i_{a}$ and $j_{b}$. Then we have

$$
w\left(i_{a}, j_{b}\right)= \begin{cases}p_{i} p_{j} \prod_{k \in \Psi\left(i_{a}, j_{b}\right)}\left(1-p_{k}\right) & \text { if } i \neq j \\ p_{i} \prod_{k \in \Psi\left(i_{a}, i_{a+1}\right)}\left(1-p_{k}\right) & \text { if } i=j\end{cases}
$$

This follows from the fact that the segment $\left(i_{a}, j_{b}\right)$ is present only in joint descriptions in which $S_{i}$ and $S_{j}$ are received and any other co-descriptor that has one or more vertices in between $i_{a}$ and $j_{b}$ is missing.

We now develop an algorithm for the rate-distortion optimized design of $K$ co-descriptors. The main idea is to fix $K-1$ co-descriptors and optimize the remaining co-descriptor with respect to the fixed co-descriptors. The algorithm then proceeds to optimize the other co-descriptors in turn one at a time till it reaches a local optimum. Optimizing a given codescriptor can be posed as a shortest path problem. We construct a weighted directed acyclic graph $G=(V, E)$, where the vertex set $V$ consists of all vertices of the original polygonal curve $\mathcal{C}=\left(v_{1}, \cdots, v_{N}\right)$. The edge set $E$ is defined by introducing a directed edge from $v_{a}$ to $v_{b}$ if and only if $a<b$. An approximating polygonal curve $S_{i}$ corresponds to a path in $G$ from $v_{1}$ to $v_{N}$, with each segment of $S_{i}$ being an edge in $E$. In the following, we will associate the rate-distortion performance of a co-descriptor $S_{i}$ to the sum of edge weights of the path corresponding to $S_{i}$.

Suppose that co-descriptor $S_{i}=\left(i_{1}, \cdots, i_{M_{i}}\right)$ is to be optimized in the next iteration. Note that all permissible segments $\left(j_{a}, k_{b}\right)$ defined on vertices of $S_{1}, \cdots, S_{i-1}, S_{i+1}, \cdots$, $S_{K}$, from the previous iteration remain in the set $\Xi$. But their weights $w\left(j_{a}, k_{b}\right)$ may be changed by the inserted vertices of $S_{i}$. We quantify these changes in $w\left(j_{a}, k_{b}\right)$ by assigning proper weights to the edges of $G$. Consider a segment $\left(i_{t}, i_{t+1}\right)$ of $S_{i}$ that has an intersection with a permissible segment $\left(j_{a}, k_{b}\right)$. There are four cases: 1. $j_{a}<i_{t}<k_{b}<i_{t+1}$; 2. $i_{t}<j_{a}<i_{t+1}<k_{b} ; 3 . j_{a}<i_{t}<i_{t+1}<k_{b} ; 4$. $i_{t}<j_{a}<k_{b}<i_{t+1}$. In case 1 , we add to edge $\left(i_{t}, i_{t+1}\right) \in$ $E$ a weight $\Delta\left(j_{a}, k_{b}\right)=d\left(j_{a}, k_{b}\right) w\left(j_{a}, k_{b}\right) p_{i}$, which is the decrement in the contribution of segment $\left(j_{a}, k_{b}\right)$ to expected distortion $\bar{D}$ by adding another co-descriptor $S_{i}$. In cases 2 and 3 , we measure the change of $w\left(j_{a}, k_{b}\right)$ by the next edge $\left(i_{t+1}, i_{t+2}\right)$ in the path corresponding to $S_{i}$ in order not to count the change twice. There will be no change to $w\left(j_{a}, k_{b}\right)$ in case 4 , because whether segment $\left(j_{a}, k_{b}\right)$ is in a joint description does not depend on $S_{i}$ and is independent of $p_{i}$.

Furthermore, we need to consider the new permissible segments induced by the vertices of $S_{i}$ and their contribution to $\bar{D}$. For an edge $\left(i_{t}, i_{t+1}\right) \in E$ we treat the two end points separately. For the newly created permissible segments $\left(i_{t}, j_{b}\right)$ starting at $i_{t}$, we set

$$
\bar{d}\left(i_{t}, j_{b}\right)=d\left(i_{t}, j_{b}\right) p_{i} p_{j} \prod_{k \in \Psi\left(i_{t}, j_{b}\right)}\left(1-p_{k}\right)
$$


We do the same for the newly created permissible segments $\left(j_{b}, i_{t+1}\right)$ ending at $i_{t+1}$. The terms $\bar{d}\left(i_{t}, j_{b}\right)$ and $\bar{d}\left(j_{b}, i_{t+1}\right)$ are the contributions of the permissible segments in question to $\bar{D}$ following the same argument used to derive (4). Also, note that for each edge $\left(i_{t}, i_{t+1}\right) \in E$, we only consider the permissible edges of forms $\left(i_{t}, j_{b}\right)$ and $\left(j_{b}, i_{t+1}\right)$ but not $\left(j_{b}, i_{t}\right)$ nor $\left(i_{t+1}, j_{b}\right)$ to avoid accounting a newly created permissible edge more than once as we will appreciate immediately when converting the problem of optimizing $S_{i}$ to one of the shortest path.

Putting everything together, and including the rate, we assign to edge $\left(i_{t}, i_{t+1}\right) \in E$ the weight:

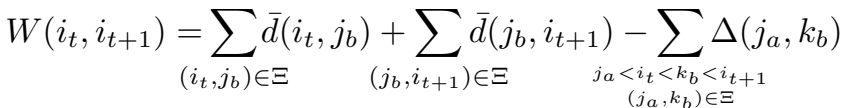

$$
\begin{aligned}
& +d\left(i_{t}, i_{t+1}\right) p_{i} \prod_{k \in \Psi\left(t_{i}, t_{i+1}\right)}\left(1-p_{k}\right)+\lambda_{i} r\left(i_{t}, i_{t+1}\right)
\end{aligned}
$$

The term $d\left(i_{t}, i_{t+1}\right) p_{i} \prod_{k \in \Psi\left(t_{i}, t_{i+1}\right)}\left(1-p_{k}\right)$ is to account for the distortion of segment $\left(i_{t}, i_{t+1}\right)$ in the event that only codescriptor $S_{i}$ is available.

Having the weights $W\left(i_{t}, i_{t+1}\right)$ defined as above for all edges in $G$, and given a co-descriptor $S_{i}$, we can see that $\sum_{1<t<M_{i}} W\left(i_{t}, i_{t+1}\right)$ measures the total change to $\bar{J}$ by adding one more co-descriptor $S_{i}$ while the other co-descriptors are fixed. Thus, minimizing $\bar{J}_{\lambda_{1}, \ldots, \lambda_{K}}\left(S_{1}, \cdots, S_{K}\right)$ over all possible $S_{i}$, given other $K-1$ co-descriptors, is equivalent to finding a shortest path from $v_{1}$ to $v_{N}$ in $G$. $G$ has $O\left(N^{2}\right)$ edges, hence the shortest path can be found in $O\left(N^{2}\right)$ time once $G$ is known. Our algorithm is summarized as follows:

1. Find a co-descriptor $S_{1}$ that minimizes $\bar{J}_{\lambda_{1}}\left(S_{1}\right)$ by finding a shortest path from $v_{1}$ to $v_{N}$ in $G$. There are no other codescriptors to be considered at this step, so the edge weights of $G$ simplify to $W\left(1_{t}, 1_{t+1}\right)=d\left(1_{t}, 1_{t+1}\right)+\lambda_{1} r\left(1_{t}, 1_{t+1}\right)$. 2. For each $i=1, \ldots, K$, find a co-descriptor $S_{i}$ that minimizes $\bar{J}_{\lambda_{1}, \ldots, \lambda_{i}}\left(S_{1}, \ldots, S_{i}\right)$ by finding a shortest path from $v_{1}$ to $v_{N}$ in $G$ with edge weights $W\left(i_{t}, i_{t+1}\right)$ as in (6).

3. Refine the co-descriptors $S_{1}, \ldots, S_{k}$ iteratively. For each $i=1, \ldots, K$, find a co-descriptor $S_{i}$ that minimizes $\bar{J}_{\lambda_{1}, \ldots, \lambda_{i}, \ldots, \lambda_{K}}\left(S_{1}, \ldots, S_{i}, \ldots, S_{K}\right)$ by finding a shortest path from $v_{1}$ to $v_{N}$ in $G$ with edge weights $W\left(i_{t}, i_{t+1}\right)$ as in (6).

4. Repeat the previous step until convergence.

It remains the problem of finding appropriate values for the Lagrange multipliers $\lambda_{1}, \ldots, \lambda_{K}$ for a given set of rate constraints on the individual co-descriptors. Since only one co-descriptor $S_{i}$ is changed at each minimization of $\bar{J}_{\lambda_{1}, \ldots, \lambda_{K}}$, the minimizing co-descriptor $S_{i}$ depends only on the Lagrange multiplier $\lambda_{i}$. We use the fast convex search method by Ramchandran and Vetterli [4] to adjust the value of $\lambda_{i}$ to the rate constraint on $S_{i}$ on each computation of $S_{i}$.

\section{VERTEX WEIGHTS AND REGIONS OF INTEREST}

So far, we assumed that all vertices of the original polygon are of equal importance and based the optimization on the distortion measure only. In some applications, it may be beneficial to assume that some vertices are more important than others. For example, in a digital map one may want to specify a certain region of interest in which the map should be more detailed. This can be achieved by assigning non-negative real valued weights to the vertices, low values indicating low importance and high values indicating high importance of the vertex. Let $\omega\left(s_{m}\right) \geq 0$ denote the weight of vertex $\mathbf{v}_{s_{m}}$. We define

$$
\hat{d}\left(s_{m}, s_{m+1}\right)=d\left(s_{m}, s_{m+1}\right) \frac{1}{s_{m+1}-s_{m}+1} \sum_{i=s_{m}}^{s_{m+1}} \omega(i)
$$

as the weighted distortion of the line segment $\left(\mathbf{v}_{s_{m}}, \mathbf{v}_{s_{m+1}}\right)$ and replace $d\left(s_{m}, s_{m+1}\right)$ by $\hat{d}\left(s_{m}, s_{m+1}\right)$ in the method described in Section 3.

\section{EXPERIMENTAL RESULTS}

In the previous sections, we did not restrict ourselves to any specific technique of coding the vertex coordinates. In our experiments, we chose a differential vertex coding scheme, which is simple and commonly used in practice with good compression performance. Specifically, a vertex $\mathbf{v}_{s_{m}}, m>0$ is predicted by the preceding vertex $\mathbf{v}_{s_{m-1}}$, and the difference vector $\delta=\mathbf{v}_{s_{m}}-\mathbf{v}_{s_{m-1}}$ is coded and transmitted. The receiver can reconstruct $\mathbf{v}_{s_{m}}$ from $\mathbf{v}_{s_{m-1}}$ by decoding $\delta$. The two components $\delta_{x}$ and $\delta_{y}$ of $\delta$ are coded in a polar coordinate system, in form of the vector length $\delta_{l}=\sqrt{\delta_{x}^{2}+\delta_{y}^{2}}$ and the angle $\delta_{\alpha}=\tan ^{-1} \delta_{y} / \delta_{x}$. The value of $\delta_{l}$ is quantized to an integer $z$, and $z$ is then encoded using a Rice code [5]. Rice coding was chosen for its low decoding complexity, which is one of our design objectives, and because the distribution of the quantized integer values $z$ is typically nearly exponential. For an exponentially distributed source, the optimal Rice parameter is given by $\left\lceil\log _{2} \mu\right\rceil$, where $\mu$ is the mean of the source. The angle $\delta_{\alpha} \in[0,2 \pi)$ is coded as a binary number in the precision of $\left\lceil\log _{2} 2 \pi z\right\rceil$ bits. Now we are ready to address an important implementation detail: Computing the rate $r\left(i_{t}, i_{t+1}\right)$ in (6) when optimizing the co-descriptor $S_{i}$. Given the Rice parameter $b_{i}$ to code $S_{i}$, the rate $r\left(i_{t}, i_{t+1}\right)$ can be easily derived for any edge. Note that the rate $r\left(i_{t}, i_{t+1}\right)$ also includes information that we need to transmit in order to specify the connectivity between the co-descriptors. For $K$ co-descriptors, we can encode the connectivity information in $K-1$ bits per line segment. For a line segment $\left(i_{t}, i_{t+1}\right)$ of co-descriptor $S_{i}$, each of the $K-1$ bits corresponds to one of the other $K-1$ co-descriptors. A bit is 1 if its co-descriptor has a vertex between $i_{t}$ and $i_{t+1}$ and 0 otherwise.

We tested out methods on a set of polygons representing country borders in a high resolution. We present results for the polygon Australia consisting of 2904 vertices (see Fig. 5 (a)). Each vertex has a precision of 32 bits in both dimensions, so the total uncompressed size of the polygon is 185856 bits.

We used the sum of the squared euclidean distances of the vertices $\mathbf{v}_{s_{m}}, \mathbf{v}_{s_{m}+1}, \ldots, \mathbf{v}_{s_{m+1}}$ to the line segment 
(a)

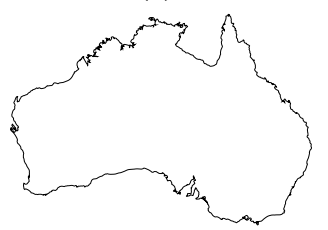

Original polygon

Fig. 1. Co-descriptors for the original polygon (a) generated for a loss probability of 0.2 and a target rate of 8192 bits: (b) regular subsampling, 186 vertices in each co-descriptor;

(c) method of Section

3, no region of interest;

(d) method of Section

3 , region of interest indicated by the box (b)
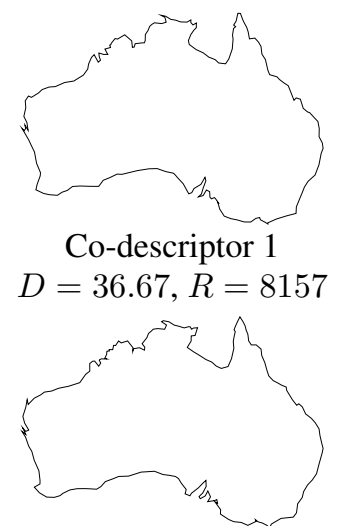

Co-descriptor 2

$D=33.39, R=8168$

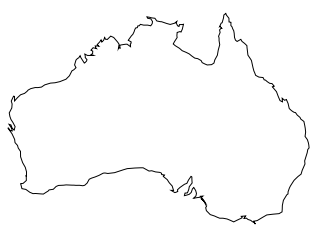

Joint descriptor

$\bar{D}=19.07$ (c)
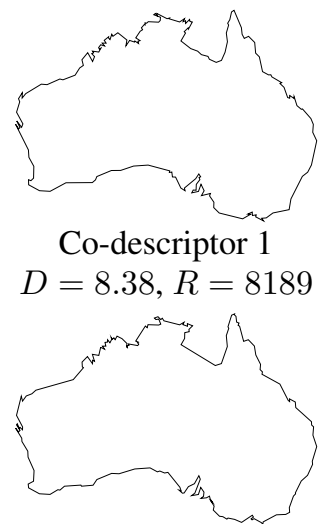

Co-descriptor 2

$D=10.85, R=8172$

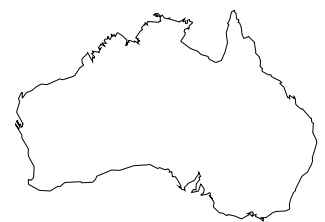

Joint descriptor

$\bar{D}=5.47$ (d)

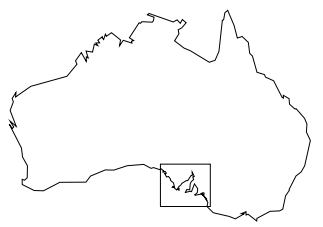

Co-descriptor 1

$D=11.25, R=8189$

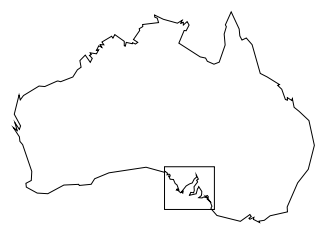

Co-descriptor 2

$D=13.35, R=8150$

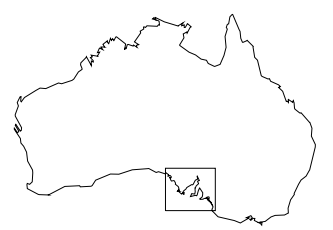

Joint descriptor

$\bar{D}=7.24$

$\left(\mathbf{v}_{s_{m}}, \mathbf{v}_{s_{m+1}}\right)$ as distortion measure $d\left(s_{m}, s_{m+1}\right)$. This distortion measure has the benefit that all distortion values can be precomputed in $O\left(N^{2}\right)$ time.

Figure 1 shows results for $K=2$. All co-descriptors were designed for a loss probability of 0.2 and a target rate of 8192 bits. The rice parameter for the vertex encoding of all descriptors was 19. $D$ is the distortion of the descriptor in units of $10^{12}, R$ is the rate of the descriptor including all necessary connectivity side information, $\bar{D}$ is the expected distortion at the receiver. The regular subsampling method shown in column (b) takes a subset of vertices which are spread uniformly over the polygon. Vertices from this subset are then assigned to the two co-descriptors in an alternating way, i.e. two consecutive vertices in the subset are assigned to different codescriptors. This method is very fast $(O(N)$ time complexity, the computation time was $240 \mathrm{~ms}$ ), but neither adaptive to the channel conditions nor to the polygon which results in a distortion much higher than the distortion of the other methods. The method of Section 3, whose results are shown in columns (c) and (d), has a higher time complexity $\left(O\left(N^{2}\right)\right.$ for one iteration) than the regular subsampling, but the obtained co-descriptors have much lower distortions. The computation times for the co-descriptors shown in columns (c) and (d) were 4.1 and 5.6 minutes, respectively. All times were measured on a $1400 \mathrm{MHz}$ Athlon-XP processor.

\section{CONCLUSION}

We proposed a new framework of multiple description geometry compression for error resilient streaming of vector graphics via lossy networks. A graph theoretical algorithm was developed to minimize the expected distortion of a co-

descriptor of an MDC code given fixed other co-descriptors. The iterative application of this algorithm enables us to construct MDC geometry codes with an arbitrary number of codescriptors that are adaptive to both, the object to compress, and the channel conditions. Experimental results validate the power of the proposed MDC algorithm in maintaining the quality of streaming graphics service in adverse channel conditions. Our approach has a very low decoding complexity. Fast and inexpensive decoding is an important advantage to many applications, particularly in wireless multimedia communications where the mobile receivers typically have limited computing resources and need to conserve battery power.

\section{REFERENCES}

[1] W. S. Chan, F. Chin, ”On approximation of polygonal curves with minimum number of line segments or minimum error", Int. J. Comput. Geom. Appl., 6, pp. 59-77, 1996.

[2] H. Imai, M. Iri, "Polygonal approximations of a curve (formulations and algorithms)", Computational Morphology, G. T. Toussaint (Ed.), pp. 71-86, NorthHolland, Amsterdam, 1988.

[3] G.M. Schuster, A.K. Katsaggelos, "An optimal polygon boundary encoding scheme in the rate distortion sense", IEEE Trans. Image Proc., vol. 7, no. 1, January 1998.

[4] K. Ramchandran, M. Vetterli, "Best wavelet packet bases in a rate-distortion sense", IEEE Trans. Image Proc., vol. 2, no. 2, pp. 160-175, April 1993.

[5] R. F. Rice, "Some practical universal noiseless coding techniques", Tech. Report, JPL-79-22, Jet Propulsion Lab, Pasadena, CA, March, 1979. 